\title{
Constrictive Pericarditis and Tuberculosis: Is It Worth More Attention?
}

\author{
Dong Wang ${ }^{1}$, Xue Sun ${ }^{2}$, and Ying Zhang ${ }^{2}$ \\ ${ }^{1}$ Affiliation not available \\ ${ }^{2}$ Shengjing Hospital of China Medical University
}

July 23, 2020

\begin{abstract}
Constrictive pericarditis (CP) characterized by the presence of a thick, fibrotic and/or calcified non-compliant pericardium limits diastolic filling and forces one side of the heart to fill at the expense of the other side. CP results in right heart failure and even reconstruction of the heart, especially for CP caused by tuberculosis (TB). As the leading cause of CP, TB is affecting the management of $\mathrm{CP}$ from many aspects, which contributes to high morbidity and mortality. The purpose of this manuscript is to: (1)Review the etiology, epidemiology, pathophysiology, diagnosis, treatment of tuberculosis constrictive pericarditis (TBCP); (2)Review animal models for CP or pericarditis through different methods; (3)Remind us that more research should be done for TBCP.
\end{abstract}

\section{KEYWORD}

Tuberculosis; Constrictive pericarditis; Model

\section{INTRODUCTION}

The pericardium is a fibroelastic sac surrounding the heart and the roots of large blood vessels. While the outer layer is a tough fibrous pericardium, the inner serous pericardium is divided into the parietal layer and the visceral layer. The parietal layer is lined on the inner surface of the fibrous pericardium, and the visceral layer adheres to the surface of the heart. The pericardial cavity is a narrow closed gap between the visceral layer and the parietal layer, which contains a small amount of slurry to play a slippery effect. The normal pericardium has a protective effect on the heart, can prevent surrounding infections from spreading to the heart, restricting the expansion of the heart and prevent the heart from rupturing quickly when the intracardiac pressure rises. CP is a disease that occurs between the visceral and parietal layers of the pericardium with a thickened and fibrosed pericardium, which may limit diastolic filling of the ventricles and cause heart failure. Constriction is the end-stage manifestation of different kinds of pericarditis, including tuberculosis pericarditis (TBP). From the perspective of CP, TB is the most known major cause. For the patients with TBP, constriction occurs in almost all untreated and many treated ones. Therefore, it is appropriate to express TBCP from two aspects: TB and constriction.

\section{ETIOLOGY AND EPIDEMIOLOGY}

The etiologic spectrum is changing all the time since CP was first described in 1669 by Lower ${ }^{[1]}$. The causes of $\mathrm{CP}$ vary by region. Although idiopathic or post-viral infection may be the leading cause of $\mathrm{CP}$ in developed countries, tubercle bacilli are still the leading cause of $\mathrm{CP}$ worldwide, especially in developing countries like Africa and a certain area in Asia. Besides, cardiac surgery, mediastinal radiation therapy, HIV and some connective tissue disorders such as systemic lupus erythematosus (SLE) and rheumatoid arthritis are also associated with $\mathrm{CP}^{[2-4]}$. TB was instigated by Mycobacterium tuberculosis (MTBC), including M. 
tuberculosis (Mtb), M. africanum, M. bovis, M.bovis Bacillus Calmette-Guerin (BCG) vaccine strain, M. canetti, M. caprae, M. pinnipedii, M. microti, and M. mungi, which infect animals as well as humans. Of these entire members of the MTBC, the M. tuberculosis, first identified in 1882 by Robert Koch, is the most infamous member, and the main reason causing TB in humans ${ }^{[5]}$.

TBP is a form of TB, which is estimated to occur in $1 \%$ to $2 \%$ of instances of pulmonary tuberculosis ${ }^{[6]}$. TBCP is one of the most severe sequelae of TBP, occurring in $30 \%$ to $60 \%$ of patients, despite prompt antiTB treatment and the use of corticosteroids ${ }^{[7]}$. Therefore, the epidemiology of TB reflects the epidemiology of TBCP to a certain extent.

It is worth mentioning that TB, one of the oldest recorded human afflictions, is still one of the biggest killers among infectious diseases, despite the extensive use of a live attenuated vaccine and several antibiotics. TB can be preserved for thousands of years in hard tissues like the bone of patients with bone TB who died more than 4000 years ago, which indicates that TB was appeared throughout the world up to then ${ }^{[8]}$. The first global estimation led by the World Health Organization (WHO) in the early 1990s reckoned that there were about 8 million incident TB cases in 1990 with 2.6 to 2.9 million deaths ${ }^{[9]}$. About 8 million new cases in 1997 and 10.4 million in 2016 ${ }^{[10]}$. The WHO's End TB Strategy ${ }^{[11]}$ have defined ambitious targets for 2035 , including a $95 \%$ reduction in the absolute number of TB deaths and a $90 \%$ reduction in TB incidence by 2035 , compared with 2015 .

The targets are far away, and we still have a long way to go. The emergence of multidrug-resistant (MDR) and extensively drug-resistant (XDR) TB makes the management of TB more difficult. MDR-TB is caused by MTBC, resistant to at least isoniazid and rifampin ${ }^{[12]}$. XDR-TB is a complicated form of MDR-TB with additional resistance to at least one kind of second-line injectable antimicrobial drug and a fluoroquinolone. It is said that approximately half a million new cases of MDR-TB challenge global health in $2017^{[10]}$, especially in India, China, and the Russian Federation ${ }^{[13]}$, with the highest-burden amongst women of reproductive age (15 to 45 years). In women of this age, tuberculosis is the leading cause of death from an infectious agent and a common non-obstetric cause of maternal mortality ${ }^{[14]}$.

The epidemic of human immune-deficiency virus (HIV) is another barrier affecting TB-control. Despite tremendous progress in the use of antiretroviral drugs for the treatment of HIV, there were still 940000 deaths and 1.8 million new infections in the world in 2017. The number of new HIV infections is increasing in about 50 countries. The total number of people living with HIV in South Africa still ranks first in the world (over 7 million), and the proportion of infected people has increased year by year, the infection rate has reached $12.57 \%{ }^{[15]}$. As is known to all, HIV and TB have a synergistic interaction. What's more, HIV causes an atypical presentation of TB with less pulmonary involvement but more extrapulmonary symptoms. It is said that TB often occurs at an earlier stage in the course of HIV infection. Pulmonary TB is the prevalent form of TB, while the count of CD4+ T lymphocytes cell is high. However, extrapulmonary TB (EPTB) becomes more prevalent, while HIV infection progresses to Acquired Immune Deficiency Syndrome $(\text { AIDS })^{[16]}$.

Another risk factor for TB that maybe forget is diabetes mellitus (DM), especially during the second half of the $20^{\text {th }}$, with the advent of widely available treatment for both TB and DM. However, the association between these two diseases has reappeared due to the global epidemic of type $2 \mathrm{DM}(\mathrm{T} 2 \mathrm{DM})^{[17]}$. According to the report published in 2017, about 415 million people live with diabetes worldwide, and an estimated 193 million people have undiagnosed diabetes. T2DM accounts for more than $90 \%$ of patients. The incidence and prevalence of DM continue to rise globally due to obesity, physical inactivity, and energy-dense diets. Besides, a meta-analysis produced evidence that smoking is a risk factor for TB infection and TB disease ${ }^{[13,18]}$.

TB is no longer a disease only caused by poverty, and the mind to end TB through reducing poverty should change. Deborah Wallace ${ }^{[19]}$ argued that debt such as housing conditions, working conditions, malnutrition, and ventilation could affect TB incidence and mortality, as well as Major social upheaval including war, mass evictions, land-grabbing, slavery, refugee-creation and denial of essential services. The Global Fund to Fight AIDS, TB, and Malaria suspended grants to North Korea, one of the highest rates of TB in the world, for 
both TB and malaria control programs on June 30, 2018. North Korea's public health system will be tested more and more in the coming years. The reasons may range from failure of the North Koreans to adhere to the Fund's standards for record-keeping to applying pressure on the North Korean regime for its nuclear endeavors. In Brazil, the election of a right-wing president, Jair Bolsonaro, who encourages land-grabbing by loggers and Big Agriculture, signals significant changes in Brazilian economic and political structure. Women's rights and the rights of marginalized populations are not guaranteed, which will destroy the system of TB control in Brazil in myriad ways. In New York, the discriminatory policy persists, such as routine denial of parole to black prisoners and routine denial of essential municipal services to poor neighborhoods, which will be detrimental to the control of TB. In Africa, feeling insecure, most working people have to work long hours for low wages under unsafe conditions, they even have no protection by culture or regulation, and then, obesity, diabetes, HIV, TB, unsafe sex, drug abuse, and violence will blossom. Therefore, we can't regard TB as only a medical problem, but it should be considered as a political and economic issue, which was driven by national interests. So not only developing nations, developed nations are not spared from this menace. When the civil war first gripped Syria in 2011, the country had a population roughly equivalent to New York state. In the eight years since the conflict has displaced more than half of its people. Liberia is within the 30 countries with the highest TB burden, probably as a consequence of the long civil war ${ }^{[20]}$. A study suggested that by 2030 , two-thirds of the world's wealth will be in the hands of $1 \%$ of the world's population. The economic and political imbalance may play an unfavorable role in the management of TB. So, it is expected that the epidemic of TBCP will go on for many years.

\section{PATHOPHYSIOLOGY}

We can understand the pathogenesis of TBCP through two main aspects-inflammation caused by tubercle bacilli and constriction of the pericardium.

Tubercle bacilli access the pericardium through 3 primary mechanisms, which include retrograde lymphatic spread from mediastinal, paratracheal and peri-bronchial lymph nodes, hematogenous spread (mainly in immunocompromised hosts), and rarely direct contiguous spread from adjacent structures such as the lungs, pleura, and spine ${ }^{[7,21]}$. The stage or progression of the disease is the result of a game between bacteria and the human immune system. Due to a competent immune system, the tuberculous pericardial disease is usually limited to the pericardial space. The immune response to the acid-fast bacilli penetrating the pericardium is associated with the incidence of TBP. Once into the pericardium, viable mycobacterial protein antigens presented by macrophages to CD4+ $\mathrm{T}$ lymphocytes trigger activation of lymphocytes, macrophages, and complement-fixing antibodies, which leads to inflammation, production of fibrinous exudate and granuloma formation. This is called delayed hypersensitivity responses induced by tubercle proteins. TBCP is the final manifestation of TBP, and there are four pathologic stages from $\mathrm{TBP}$ to $\mathrm{TBCP}^{[22]}$ : (1) fibrinous exudation with initial polymorphonuclear leukocytosis, relatively abundant mycobacteria, and early granuloma formation with the loose organization of macrophages and T cells; (2) serosanguineous effusion with a predominantly lymphocytic exudate with monocytes and foam cells; (3) effusion absorption with the organization of granulomatous caseation and pericardial thickening caused by fibrin, collagenosis, and ultimately, fibrosis; and (4) constrictive scarring, which involves fibrosis of the visceral and parietal pericardium. The scarred (and sometimes calcified) pericardium encases the heart in a fibrocalcific skin that impedes diastolic filling and causes the classic constrictive pericarditis syndrome. (Figure.2)

When TB is linked to AIDS, some things become different. In HIV-infected hosts, tubercle bacilli spread to the pericardium mainly via a hematogenous route ${ }^{[23]}$. Dysfunctional immunity in HIV/AIDS patients, EPTB such as TBP, becomes more frequent as part of a disseminated process associated with TB bacteremia accompanied by active mycobacterial replication and high bacterial load. HIV alters the natural history and outcomes of TB pericarditis. Fewer granulomas were observed than in non-HIV patients because of severely depleted CD4+ T lymphocytes ${ }^{[24]}$. Immunocompromised participants appear less likely to develop constrictive pericarditis but have significantly higher mortality compared with their immunocompetent counterparts ${ }^{[13]}$. However, when CP present in HIV-positive patients is usually due to tubercle bacilli ${ }^{[25]}$.

It seems to be a fundamental difference in the susceptibility between HIV patients and T2DM patients to 
$\mathrm{TB}^{[26]}$. Macrophage function is altered during T2DM in tubercle bacilli-naïve individuals, which include reducing binding or phagocytosis of tubercle bacilli ${ }^{[27]}$. Chronic inflammation and dysregulation of the inflammasome, a common immunological characteristic to obesity and T2DM, may be associated with the increased susceptibility to $\mathrm{TB}^{[28]}$. What's more, tubercle bacilli can reside and persist in adipose tissues, and evade recognition by the host immune system so that forming a reservoir for possible re-activation ${ }^{[29]}$. However, the relationship between T2DM and TB still mostly unknown.

Except for some pathological factors above, pregnancy can also make the management of TB more difficult. For pregnant women, the increased blood volume during pregnancy is undoubtedly worse for the scarred heart. In women co-infected with tuberculosis and HIV, neonates born to these women also have increased morbidity and mortality ${ }^{[30]}$.

The restriction is another property of TBCP. As is known to all, the pericardial space contains up to $50 \mathrm{ml}$ of plasma ultrafiltrate, which can reduce friction on the epicardium and equalizes gravitational, hydrostatic, and inertial forces over the surface of the heart, so that transmural cardiac pressures neither change during acceleration nor differ regionally within cardiac chambers. Besides, pericardial reserve such as oblique sinus, transverse sinus, and pericardial recesses, together with the histological components of pericardium like collagen and short elastin fibers neutralize the effects of respiration and change of body position ${ }^{[31]}$. When inhaling, the intrathoracic pressure decreases, and this pressure difference is transmitted into the heart chambers so that the pressure changes in the heart and intrathoracic are synchronous. When the restriction occurs, a thickened, inelastic, and even calcified pericardium limits diastolic filling ${ }^{[32]}$ and prevents the regular inspiratory decrease in intrathoracic pressure from being transmitted to the heart chambers, causing dissociation of intracardiac and intrathoracic forces. Since the pericardium does not surround the pulmonary veins, so the pulmonary venous pressure but not left atrium (LA) pressure usually drops during inspiration, which causes a reduction of flow from pulmonary venous into LA, and then a reduction in left ventricle (LV) volume ${ }^{[33]}$. With this slight reduction in LV filling, the interventricular septum bows to the left and right ventricle (RV) filling increases slightly. (Figure.3) The opposite effects occur during expiration due to ventricular interdependence. Subsequently, intracardiac pressures rise, and there is eventual equalization of diastolic pressures. Although the systolic ventricular function is reasonable, the impaired ventricular filling will lead to a reduction of stroke volumes and cardiac output ${ }^{[34]}$. The restricted pericardium can also cause the right-side heart failure (HF) of patients with symptoms of edema, hepatomegaly, and effusion in serous cavities develop ${ }^{[35]}$. To make matters worse, TBCP is often accompanied by calcification. As the disease progresses, calcification may penetrate the myocardium, leading to remodeling of the heart structure. As a result, the mortality rate increases.

\section{DIAGNOSIS}

\section{History}

The patient's medical histories, including history and present history, are crucial for the Diagnosis of TBCP, especially for the patients with an obvious constriction symptom and unclear etiology. When the constriction is the first symptom, whether the patient is in an epidemiologically high-risk area of TB and/or HIV has a tremendous, supportive effect on the diagnosis. At the same time, we know that many factors/diseases can lead to CP, such as uremia, SLE, rheumatoid arthritis, cardiac surgery, mediastinal radiotherapy, and viral infection, etc. These related factors/diseases are also helpful for differential diagnosis. Moreover, such clinical features like low fever, night-sweats, and weight-loss should be valued.

\section{Laboratory assessment}

Notable elevations of IL-10 and IFN- $\gamma$, together with a low level of bioactive TGF- $\beta$ in tuberculous pericardial fluid, indicate delayed-type hypersensitivity response to the pathogen mediated by Th- $1^{[36]}$. Elevated pericardial adenosine deaminase (ADA) activity and lysozyme levels are essential in the Diagnosis of TBP ${ }^{[37]}$. It is said that a pericardial ADA level[?]40U/L has a sensitivity and specificity of $88 \%$ and $83 \%$, respectively ${ }^{[38]}$. However, it is not always possible to obtain pericardial fluid or tissue, especially when the constriction has occurred. In the endemic area, globulin level higher than $40 \mathrm{~g} / \mathrm{L}$, and peripheral leukocyte count less than 
$10 \times 10^{\wedge} 9 \mathrm{~g} / \mathrm{L}$ may infer an infection of TB. Besides, the positive rates of procalcitonin (PCT), C-reactive protein (CRP) are high in the serum of patients with TB. And the measurement of plasma brain natriuretic peptide (BNP) level-a marker of ventricular dysfunction and wall stretch-may be useful in the Diagnosis of CP.

\section{Physical examination}

The classic presentation of TBCP is of right-sided heart failure-hepatomegaly, ascites, and leg edema ${ }^{[39]}$. Patients with CP typically have markedly elevated central venous pressure, and the jugular venous pressure may also rise with inspiration (Kussmaul sign). The systolic blood pressure may reveal an abnormal ( $>10$ $\mathrm{mm} \mathrm{Hg}$ ) decrease while inspiration, which is termed 'pulses paradoxus' and relates to the dissociation of intrathoracic and intracardiac pressures ${ }^{[40]}$. Besides, symptoms, such as weakness, cough, chest pain, night sweats, fever, and tachycardia may be found.

\section{Electrocardiography}

Electrocardiogram (ECG) is a technology that records the changes in cardiac electrical activity during each cardiac cycle from the body surface ${ }^{[41]}$. It can not only reflect the changes in the heart rhythm, but also has a prompting effect on pericarditis, especially for patients with $\mathrm{CP}$ who are not accompanied by pericardial thickening or calcification. Morris first introduced the P-wave terminal force (PTF) in lead V1 (PTFV1) measured by 12-lead ECG that can reflect left atrial structural abnormalities and diastolic dysfunction of left ventricular ${ }^{[42]}$. Yanhong Ren's study ${ }^{[43]}$ indicated a close association of abnormal PTFV1([?]-0.04mm $\left.\cdot \mathrm{s}\right)$ and $\mathrm{TBCP}$, which could remedy the limitation of X-ray, CT, and echocardiography examination in the TBCP diagnosis. The abnormality of PTFV1 may associated with the load increasing of the left atrial and the right atrial, leading to an prolongment of the left atrial depolarization and the interatrial bundle conduction interval. What's more, low voltage and atrial fibrillation may be present in part of patients with CP.

\section{Echocardiogram}

The American College of Cardiology and the European Society of Cardiology guidelines recommend the use of echocardiography for diagnosis of constrictive pericarditis and any other pericardial disease.

(1)Increased pericardial thickness with or without calcification may be reflected in two-dimensional echocardiography.

(2)The shift of ventricular septum may be observed in CP with a sensitivity of $93 \%{ }^{[44]}$.

(3)Comparing with an average person, armored pericardium contributes to the dissociation of intracardiac and intrathoracic pressures, which causes a reduction in mitral early (E) inflow velocities during inspiration.

(4)Due to the high right atrium (RA) pressure, the resistance from hepatic vein to RA increases, and expiration will undoubtedly enlarge the obstruction. So hepatic vein diastolic flow reversal during expiration is a specific (88\%) sign of $\mathrm{CP}$.

(5)Tissue Doppler, which may have a preserved or elevated e', is often used to evaluate diastolic function. Also, we can see the medial e' velocity is higher than the lateral of the myocardium, which is exactly the opposite of routine, and called 'annulus reverses' ${ }^{[45]}$.

(6)Two-dimensional speckle tracking echocardiography aiming at evaluating myocardial mechanics provide additional diagnostic and clinical information in the context of CP. The typical longitudinal deformation pattern of $\mathrm{CP}$ includes preserved septal and reduced longitudinal strain values in left ventricular free wall myocardial segments due to pericardial adhesions, which is different from restrictive cardiomyopathies-where longitudinal strain is usually uniformly reduced, and the values are much lower than $\mathrm{CP}^{[46,47]}$.

\section{Computed tomography}

Computed tomography (CT) is not a common examination for heart disease, however, it can show pericardial calcification sensitively, and show the relation-adhesion or not-between pericardium and tissues surrounding 
the heart. Therefore, CT is helpful in patients evaluating before pericardiectomy and making operative planning ${ }^{[31]}$. Besides, CT can find pulmonary tuberculosis, which is helpful for the Diagnosis of TBCP.

\section{Cardiac magnetic resonance imaging}

Cardiac magnetic resonance imaging (CMR) is a common tool for assessing pericardial diseases. Not only anatomical information but ventricular septal motion inferring constrictive physiology can also be reflected via $\mathrm{MRI}^{[48]}$.

The alterations of $\mathrm{CP}$ in the CMR mainly has the following points: (1)pericardial thickness equal or larger than 4mm; (2)dilatation of the vena cava and supra-hepatic veins which represents the high pressure of RA; (3)delayed gadolinium enhancement of the pericardium is associated with increased fibroblast proliferation and neovascularization, as well as more prominent chronic inflammation and granulation tissue ${ }^{[49,50]}$.

\section{Positron emission tomography/computed tomography}

Positron emission tomography/computed tomography (PET/CT), which is an image fusion about PET and $\mathrm{CT}$, can provide both anatomy structure and function information. ${ }^{18} \mathrm{~F}$-2-deoxy-2-fluoro-D-glucose $\left({ }^{18} \mathrm{~F}\right.$ FDG), which can reflect glucose metabolism and find the abnormal part, is the common tracer of PET/CT. Although ${ }^{18}$ F-FDG PET/CT is commonly used in oncology fields, it can also visualize the inflammation. Activated inflammatory cells predominantly metabolize glucose as a source of energy. Even more, we often use it to differentiate pulmonary TB from lung cancer. The degrees of FDG uptake in the pericardium and the mediastinal and supraclavicular lymph nodes are useful for differentiating acute tuberculous from idiopathic pericarditis ${ }^{[51]}$. TBCP is frequently associated with pulmonary TB or lymph node involvement, which can be detected by ${ }^{18} \mathrm{~F}$-FDG PET/CT-one of the advantage of it. Diagnosis is made on a multifactorial analysis of clinical, imaging, chemical, and bacteriological and serological basis, although PET/CT has a high sensitivity but low specificity ${ }^{[52]}$. However, molecular imaging has great potential, which depends on the development and selection of tracers. Not only diagnosis/differential diagnosis but also the stage of the disease can be made, as well as an evaluation of the treatment effect.

\section{Biopsy}

A definite and direct approach to diagnose TBP involves identifying acid-fast bacilli (AFB) in any body site. But the long waiting time limits its value in clinical decision. A pericardial biopsy may be helpful for diagnosing TBP. Typical histology with caseous necrosis and granulomatous inflammation or positive AFB staining can confirm the Diagnosis. However, when the progression comes to the end-stage, typical microscopy findings can disappear, and left only fibrotic changes and chronic inflammation. So the Diagnosis of TBCP should be made early or the etiology of the disease should be clear at the early stage. Once the Diagnosis of the restriction has been made, establishing a TB etiology is less straight forward, for a large proportion of patients present restriction but have no prior history of $\mathrm{TB}^{[53,54]}$. So the response to anti-tuberculosis treatment should be another criterion of the Diagnosis of TBCP.

The most challenging aspect of the Diagnosis of TBCP (Figure.4) remains to be establishing a tubercle bacilli etiology, especially in countries in which TB is not endemic. For those patients with TBCP who developed from TBP, the Diagnosis is relatively simple. Although there are four stages of TB (dry, effusive, absorptive, and constrictive), TBCP may not always result from the progression of acute pericarditis from a dry stage sequentially through an effusive, and absorptive, but may mainly result from a fibrotic pericardium without previous history of acute pericarditis. What's more, the clinical presentation of constriction is highly variable, which may range from asymptomatic to severe constriction. When the disease has reached the advanced stage and the constriction has formed, finding the etiology may be hard, for the typical caseous necrosis has disappeared. Therefore, whether in the endemic area and history are vital to the Diagnosis of TBCP. Pericarditis-related diseases or procedures such as TB, HIV, T2DM, obesity, chest radiotherapy, SLE, uremia, infecting by viral, and a history of heart surgery and so on should be mastered, which can help us make a faster and accurate diagnosis. Also, we should pay attention to the differences in the Diagnosis of TBCP accompanied by HIV or T2DM and TBCP caused by MDR-TB or XDR-TB. 


\section{TREATMENT}

The treatment of TBCP is aimed at achieving three goals: killing and control of active Mtb; relief of the cardiac compression due to the fibrosed pericardium; and the prevention of complications or adverse reactions. Among these, removing fibrosed pericardium is much more urgent. Four anti-tuberculosis drug regimens (rifampicin, isoniazid, ethambutol, and pyrazinamide) for a minimum of six months, killing and controlling Mtb effectively, has been the standard care of TB for decades. The drugs can easily reach the lesion to take effect through the mouth, due to the abundant blood vessels in the lungs, which is different from the pericardial cavity. A recent study found that the penetration of anti-tuberculosis drugs in the pericardial space is low during the first 24 hours following drug administration. The median peak value of rifampicin concentrations in the pericardial fluid was significantly lower than the minimum inhibitory concentration, and isoniazid was the only drug with sufficient concentration found in pericardial fluid in patients with $\mathrm{TBP}^{[55]}$. This is a study of patients with TBP manifesting as pericardial effusion. However, while it progresses to TBCP with fibrosis gradually forming and blood vessels decreasing, the effect of chemotherapy will become smaller, especially following the formation of calcification. Therefore, the only definite treatment of TBCP is surgical pericardiectomy, but it has a high perioperative mortality of between $5 \%-14 \%{ }^{[53]}$ and optimistic prognosis. Considering that TBCP may not exist alone, and it may be accompanied by tuberculosis lesions in other parts of the body, anti-tuberculosis treatment is still necessary. Some researchers had achieved good results with pure anti-tuberculosis drugs for TBCP, but it seems to be related to the stage of the disease, and there is less research to support such results. It's helpful for prognosis by taking pericardiectomy timely when the anti-tuberculosis does not work. However, the timing of pericardiectomy is controversial. Some authors suggested that we should use standard anti-TB drugs for 6 months and then pericardiectomy for persistent constriction in the face of drug therapy. Some recommended pericardiectomy should be done if the patient's condition is static hemodynamically or deteriorates after 4 to 8 weeks of anti-TB therapy. Also some though surgery should be undertaken earlier under anti-TB therapy if the pericardial calcification appears $^{[22]}$. There is no consensus on the scope of a pericardiectomy for the surgical treatment of chronic constrictive pericarditis. A total pericardiectomy is technically more demanding but has superior resultsboth early and late compared with partial pericardiectomy ${ }^{[56]}$. The process of pericardiectomy is difficult, especially with calcification penetrating the myocardium, which may damage myocardial laceration and endanger life during operation ${ }^{[57]}$. At the same time, diuretic therapy, inotropic support and some device support such as cardiopulmonary bypass (CPB), ultrasonic bone scalpel, and extracorporeal membrane oxygenation (ECMO) may be needed during or after the pericardiectomy. Besides, advanced age, atrial fibrillation, tricuspid insufficiency, need for inotropic support, low cardiac output, high atrial pressure, ascites, low functional status and the duration of constriction have been related to poor outcomes ${ }^{[56]}$.

As for MDR-TB or XDR-TB, chemotherapy drugs need some adjustments, using second-line drugs instead of the four drugs. Using only amikacin in shorter regimens and bedaquiline or linezolid in longer regimens were recommended ${ }^{[58]}$. Drug susceptibility testing (DST) is necessary for the treatment of patients with Mtbpositive. Since little is known about the use of second-line tuberculosis drugs in pregnancy, the management of MDR/XDR-TB in pregnant women is more complicated. And the use of new and repurposed drugs during pregnancy and breastfeeding is not widely recommended. Given the complexity of treating pregnant women with MDR/XDR-TB, individualized treatment should be done for each one from hospitalizing. Aggressive treatments should be used under safety as the mortality of pregnancy-associated TB can up to $40 \%$.

The infection of HIV of patients with TBCP increases the mortality rate greatly. However, the optimal therapeutic strategy concerning the duration of anti-TB therapy is uncertain. Concern about the potential for the development of toxicities, side effects, immune reconstitution syndrome (IRIS) and complex drugdrug interactions if ART is introduced early during therapy for TB often led to long delays. It is said that the use of corticosteroids may prevent the development of TBCP from TBP in the patient without HIV ${ }^{[59]}$. However, whether corticosteroid is beneficial for the treatment of TBCP still needs further studies. What is certain is that corticosteroid potentially causes complications, including aggravation of diabetes, Cushingoid features, infection risk, osteoporosis and increasing cancer risk for HIV/AIDS patients ${ }^{[33]}$. And the role of pericardiectomy still needs further exploration. 


\section{ANIMAL MODEL}

A suitable animal model can be used to test key hypotheses at a much faster rate than the research based on the patient. In clinical researches, we have to measure something related to the desired variable, with low credibility, due to an impractical or impossible measurement of the desired variable. Thus, a reasonable animal model represents a remarkably powerful approach for the solution of clinical problems ${ }^{[60]}$. TBCP has the characteristics of difficulty in Diagnosis, untimely discovery, and high mortality. Considering these aspects and the global epidemic of TB and the related diseases, we suggest that a reasonable animal model of TBCP is necessary. So far, no researches of animal model about TBCP have been found. Some animal models of pericarditis and other kinds of $\mathrm{CP}$ are shown in the table below. (Table.1)

\section{DISCUSSION}

CP may be caused by various factors, some of which may be transient, while TBCP may manifest as a progressive constriction even treated. Although idiopathic, post-cardiac surgery and radiation were the three most common etiologies of CP in some developed countries. Considering the high incidence of TBP in developing countries, the prevalence of MDR-TB, the impact of HIV, T2DM, obesity and some national policies, TB will still be the main cause of CP for a long time worldwide.

Constriction is the end-stage manifestation of TBP, which contributes to the death of patients with TBP. Early Diagnosis and treatment of TBCP are crucial for prognosis. As we have known that TBP has four pathological stages-dry, effusive, absorptive, and constrictive. However, many patients with TBCP who give no history of an acute attack have in fact had such an attack, which has been overlooked because of the mildness of the constitutional symptoms. For these patients, it is still controversial whether the constriction results from the progression of acute pericarditis from a dry stage sequentially through an effusive, absorptive and constrictive phase ${ }^{[71,72]}$. Moreover, when it progresses to chronic constriction, the etiologic Diagnosis may be more impossible and the treatment only left pericardiectomy. Limited by the fibrosis pericardium for a long time, the myocardial function may be impaired. The perioperative mortality rate is high even with extracorporeal circulation while calcification has penetrated the myocardium causing a reconstruction of the heart.

To date, little progress has made in our understanding of the immunopathogenesis of TBCP, especially accompanied by HIV, T2DM, and obesity. Neither a relatively inexpensive, rapid, accurate, and widely available test has been developed, nor the determinants and drivers of TBCP have been understood, much less is the pharmacokinetics and pharmacodynamics of anti-tuberculous drugs in both plasma and the pericardium and their impact on short- and long-term outcomes. Therefore, there seems to be a long way to go ${ }^{[73]}$.

\section{Acknowledgements}

We wish to acknowledge the support of Professor Wei-dong Ren, Chair of our Department, for his great assistance in this study.

\section{Authors' contributions}

DW conceived study. XS analyzed the echocardiograms. DW developed the first draft of the manuscript. XS helped revise the manuscript. YZ helped revise the manuscript and provided with oversight to the project. All authors read and approved the final manuscript.

\section{REFERENCE}

1. Lower R: Treatment of Heart[in Latin]. London: Allestry;1669.

2. Wang FF, Hsu J, Jia FW, et al: Left ventricular strain is associated with acute postoperative refractory hypotension in patients with constrictive pericarditis and preserved ejection fraction. J Thorac Dis 2018:10(7):4147-4155.

3. Pessinaba S, Sonhaye L, Agbétiafa M, et al: The chronic constrictive pericarditis, a real calcified gangue realizing a mid-ventricular bottleneck: A case report. Ann Cardiol Angeiol(Paris) 2019:68(2):125-128. 
4. Albarrán AA, González JAB, García JMM: "Malignant" Chronic Constrictive Pericarditis. Eurasian J Med 2018:50(2):140.

5. Bhembe NL, Green E: Molecular epidemiological study of multidrug-resistant tuberculosis isolated from sputum samples in Eastern Cape, South Africa. Infect Genet Evol 2020:80:104182.

6. Fowler NO: Tuberculous pericarditis. JAMA 1991:266(1):99-103.

7. Ntsekhe M, Mayosi BM: Tuberculous pericarditis with and without HIV. Heart Fail Rev 2013:18(3):367373.

8. Smith I: Mycobacterium tuberculosis pathogenesis and molecular determinants of virulence. Clin Microbiol Rev 2003:16(3):463-496.

9. Sudre P, ten Dam G, Kochi A: Tuberculosis: a global overview of the situation today. Bull World Health Organ 1992:70(2):149-159.

10. World Health Organization: Global tuberculosis report 2019 WHO/CDS/TB/2019.15. Geneva; 2019.

11. World Health Organization: The end TB strategy. Geneva, Switzerland: 2015.

12. Walker TM, Merker M, Knoblauch AM, et al: A cluster of multidrug-resistant Mycobacterium tuberculosis among patients arriving in Europe from the Horn of Africa: a molecular epidemiological study. Lancet Infect Dis 2018:18(4):431-440.

13. Glaziou P, Floyd K, Raviglione MC: Global Epidemiology of Tuberculosis. Semin Respir Crit Care Med 2018:39(3):271-285.

14. Loveday M, Hughes J, Sunkari B, et al: Maternal and infant outcomes among pregnant women treated for multidrug/rifampicin-resistant tuberculosis in South Africa. Clin Infect Dis 2020:ciaa189.

15. UNAIDS: Global AIDS update 2018[M]. Geneva: UNAIDS, 2018:12-18.

16. Mastroianni A, Coronado O, Chiodo F: Tuberculous pericarditis and AIDS: case reports and review. Eur J Epidemiol 1997:13(7):755-759.

17. Mtabho CM, Semvua HH, van den Boogaard J, et al: Effect of diabetes mellitus on TB drug concentrations in Tanzanian patients. J Antimicrob Chemother 2019:74(12):3537-3545.

18. Bates MN, Khalakdina A, Pai M, et al: Risk of tuberculosis from exposure to tobacco smoke: a systematic review and meta-analysis. Arch Intern Med 2007:167(4):335-342.

19. Wallace D, Wallace R: Problems with the WHO TB model. Math Biosci 2019:313:71-80.

20. López MG, Dogba JB, Torres-Puente M, et al: Tuberculosis in Liberia: high multidrug-resistance burden, transmission and diversity modelled by multiple importation events. Microb Genom 2020:6(1):e000325.

21. SPODICK DH: Tuberculous pericarditis. AMA Arch Intern Med 1956:98(6):737-749.

22. Mayosi BM, Burgess LJ, Doubell AF: Tuberculous pericarditis. Circulation 2005:112(23):3608-3616.

23. Syed FF, Mayosi BM: A modern approach to tuberculous pericarditis. Prog Cardiovasc Dis 2007:50(3):218-236.

24. Reuter H, Burgess LJ, Schneider J, et al: The role of histopathology in establishing the Diagnosis of tuberculous pericardial effusions in the presence of HIV. Histopathology 2006:48(3):295-302.

25. Hakim JG, Ternouth I, Mushangi E, et al: Double blind randomised placebo controlled trial of adjunctive prednisolone in the treatment of effusive tuberculous pericarditis in HIV seropositive patients. Heart 2000:84(2):183-188.

26. Ronacher K, Joosten SA, van Crevel R, et al: Acquired immunodeficiencies and tuberculosis: focus on HIV/AIDS and diabetes mellitus. Immunol Rev 2015:264(1):121-137.

27. Gomez DI, Twahirwa M, Schlesinger LS, et al: Reduced Mycobacterium tuberculosis association with monocytes from diabetes patients that have poor glucose control. Tuberculosis 2013:93(2):192-197.

28. Tack CJ, Stienstra R, Joosten LA, et al: Inflammation links excess fat to insulin resistance: the role of the interleukin-1 family. Immunol Rev 2012:249(1):239-252.

29. Neyrolles O, Hernández-Pando R, Pietri-Rouxel F, et al: Is adipose tissue a place for Mycobacterium tuberculosis persistence?. PLoS One 2006:1(1):e43.

30. Mathad JS, Gupta A: Tuberculosis in Pregnant and Postpartum Women: Epidemiology, Management, and Research Gaps. Clinical Infectious Diseases 2012:55(11):1532-1549.

31. Klein AL, Abbara S, Agler DA, et al: American Society of Echocardiography clinical recommendations 
for multimodality cardiovascular imaging of patients with pericardial disease: endorsed by the Society for Cardiovascular Magnetic Resonance and Society of Cardiovascular Computed Tomography. J Am Soc Echocardiogr 2013:26(9):965-1012.

32. Adler Y, Charron P, Imazio M, et al: 2015 ESC Guidelines for the Diagnosis and management of pericardial diseases: The Task Force for the Diagnosis and Management of Pericardial Diseases of the European Society of Cardiology (ESC)Endorsed by: The European Association for Cardio-Thoracic Surgery (EACTS). Eur Heart J 2015:36(42):2921-2964.

33. Hoit BD: Pathophysiology of the Pericardium. Prog Cardiovasc Dis 2017:59(4):341-348.

34. Tuck BC, Townsley MM: Clinical Update in Pericardial Diseases. J Cardiothorac Vasc Anesth 2019:33(1):184-199.

35. D'Elia E, Ferrazzi P, Imazio M, et al: Constrictive pericarditis: a common pathophysiology for different macroscopic anatomies. J Cardiovasc Med (Hagerstown) 2019:20(10):725-726.

36. Ntsekhe M, Matthews K, Syed FF, et al: Prevalence, hemodynamics, and cytokine profile of effusiveconstrictive pericarditis in patients with tuberculous pericardial effusion. PLoS One 2013:8(10):e77532.

37. Reuter H, Burgess L, van Vuuren W, et al: Diagnosing tuberculous pericarditis. QJM 2006:99(12):827839.

38. Tuon FF, Litvoc MN, Lopes MI: Adenosine deaminase and tuberculous pericarditis-a systematic review with meta-analysis. Acta Trop 2006:99(1):67-74.

39. Miranda WR, Oh JK: Constrictive Pericarditis: A Practical Clinical Approach. Prog Cardiovasc Dis 2017:59(4):369-379.

40. Welch TD: Constrictive pericarditis: Diagnosis, management and clinical outcomes. Heart 2018:104(9):725-731.

41. Maron BJ, Friedman RA, Kligfield P, et al: Assessment of the 12-lead ECG as a screening test for detection of cardiovascular disease in healthy general populations of young people (12-25 Years of Age): a scientific statement from the American Heart Association and the American College of Cardiology. Circulation 2014:130(15):1303-1334.

42. Morris JJ Jr, Estes EH Jr, Whalen RE, et al: P-wave analysis in valvular heart disease. Circulation 1964:29:242-252.

43. Ren Y, Qiu J, Li Z, et al: P-wave terminal force in lead V1 is a predictive indicator for the Diagnosis of tuberculous constrictive pericarditis. Heart Lung 2019:48(2):155-158.

44. Welch TD, Ling LH, Espinosa RE, et al: Echocardiographic Diagnosis of constrictive pericarditis: Mayo Clinic criteria. Circ Cardiovasc Imaging 2014:7(3):526-534.

45. Reuss CS, Wilansky SM, Lester SJ, et al: Using mitral' annulus reversus' to diagnose constrictive pericarditis. Eur J Echocardiogr 2009:10(3):372-375.

46. Jottrand E, Serste T, Mulkay JP, et al: Longitudinal strain by speckle tracking echocardiography in constrictive pericarditis. Eur Heart Cardiovasc Imaging 2018:19(6):638.

47. Madeira M, Teixeira R, Costa M, et al: Two-dimensional speckle tracking cardiac mechanics and constrictive pericarditis: systematic review. Echocardiography 2016:33(10):1589-1599.

48. Anavekar NS, Wong BF, Foley TA, et al: Index of biventricular interdependence calculated using cardiac MRI: a proof of concept study in patients with and without constrictive pericarditis. Int J Cardiovasc Imaging 2013:29(2):363-369.

49. Carrascosa PM, Deviggiano A, Rodríguez-Granillo GA: Pericardial Disease. Clinical Atlas of Cardiac and Aortic CT and MRI[M]. Switzerland: Springer,2019:309-326.

50. Zurick AO, Bolen MA, Kwon DH, et al: Pericardial delayed hyperenhancement with CMR imaging in patients with constrictive pericarditis undergoing surgical pericardiectomy: a case series with histopathological correlation. JACC Cardiovasc Imaging 2011:4(11):1180-1191.

51. Dong A, Dong H, Wang Y, et al: (18)F-FDG PET/CT in differentiating acute tuberculous from idiopathic pericarditis: preliminary study. Clin Nucl Med 2013:38(4):e160-e165.

52. Kim MS, Kim EK, Choi JY, et al: Clinical Utility of [18F]FDG-PET /CT in Pericardial Disease. Curr Cardiol Rep 2019:21(9):107.

53. Mutyaba AK, Ntsekhe M: Tuberculosis and the Heart. Cardiol Clin 2017:35(1):135-144. 
54. Chang SA: Tuberculous and Infectious Pericarditis. Cardiol Clin 2017:35(4):615-622.

55. Shenje J, Ifeoma Adimora-Nweke F, Ross IL, et al: Poor Penetration of Antibiotics Into Pericardium in Pericardial Tuberculosis. EBioMedicine 2015:2(11):1640-1649.

56. Metaxas EI, Kotsifas K, Tatsis G, et al: Tuberculous pericarditis: three cases and brief review. Monaldi Arch Chest Dis 2010:73(1):44-50.

57. Ak K, Demirbas E, Atas H, et al: Results of pericardiectomy for constrictive pericarditis: Single-center experience. Herz 2017:42(1):75-83.

58. Falzon D, Schünemann HJ, Zignol M, et al: WHO guidance on multidrug-resistant tuberculosis treatment: using and communicating the evidence. Eur Respir J 2020:55(3):1902325.

59. World Health Organization: Guidelines for treatment of drug susceptible tuberculosis and patient care, 2017 update. Geneva, Switzerland: WHO, 2017.

60. Klopfenstein HS: The pathophysiology of pericardial disease-contributions derived from echocardiography/Doppler studies in animal models. Echocardiography 1990:7(1):5-9.

61. Tsui CY, Burch GE: Coxsackie virus B4 pericarditis in mice. Br J Exp Pathol 1971:52(1):47-50.

62. Matsumori A, Kawai C: Coxsackie virus B3 perimyocarditis in BALB/c mice: experimental model of chronic perimyocarditis in the right ventricle. J Pathol 1980:131(2):97-106.

63. Reeves WC, Cunningham D, Schwiter EJ, et al: Myocardial hydroxyproline reduced by early administration of methylprednisolone or ibuprofen to rabbits with radiation-induced heart disease. Circulation 1982:65(5):924-927.

64. Pandian NG, Skorton DJ, Kieso RA, et al: Diagnosis of constrictive pericarditis by two-dimensional echocardiography: studies in a new experimental model and in patients. J Am Coll Cardiol 1984:4(6):1164-1173.

65. Pagé PL, Plumb VJ, Okumura K, et al: A new animal model of atrial flutter. J Am Coll Cardiol 1986:8(4):872-879.

66. Leak LV, Ferrans VJ, Cohen SR, et al: Animal model of acute pericarditis and its progression to pericardial fibrosis and adhesions: ultrastructural studies. Am J Anat 1987:180(4):373-390.

67. Afanasyeva M, Georgakopoulos D, Fairweather D, et al: Novel model of constrictive pericarditis associated with autoimmune heart disease in interferon-gamma-knockout mice. Circulation 2004:110(18):29102917.

68. d'Avila A, Neuzil P, Thiagalingam A, et al: Experimental efficacy of pericardial instillation of antiinflammatory agents during percutaneous epicardial catheter ablation to prevent postprocedure pericarditis. J Cardiovasc Electrophysiol 2007:18(11):1178-1183.

69. Jiamsripong P, Alharthi MS, Calleja AM, et al: Quantification of left ventricular twisting mechanics by velocity vector imaging in an animal model of pericardial adhesions. Ultrasound Med Biol 2009:35(12):1963-1972.

70. Bokeriya LA, Bokeriya OL, Sivtsev VS, et al: Experimental Evaluation of Biodegradable Film Compositions Based on Gelatin with Colchicine. Bull Exp Biol Med 2016:161(3):414-418.

71. Tse G, Ali A, Alpendurada F, et al: Tuberculous Constrictive Pericarditis. Res Cardiovasc Med 2015:4(4):e29614.

72. Kim MS, Chang SA, Kim EK, et al: The Clinical Course of Tuberculous Pericarditis in Immunocompetent Hosts Based on Serial Echocardiography. Korean Circ J 2020:50(7):599-609.

73. Isiguzo G, Du Bruyn E, Howlett P, et al: Diagnosis and Management of Tuberculous Pericarditis: What Is New?. Curr Cardiol Rep 2020:22(1):2.

\section{Figure legends}

\section{Figure.1 Mind Mapping}

This is the mind mapping of this review, and the review will introduce one by one according to the part of the mind mapping. $\mathrm{TBCP}=$ tuberculous constrictive pericarditis, $\mathrm{TB}=$ tuberculosis, $\mathrm{MDR}=$ multidrugresistant, $\mathrm{XDR}=$ extensively drug-resistant, $\mathrm{HIV}=$ human immune-deficiency virus, $\mathrm{T} 2 \mathrm{DM}=$ type 2 diabetes mellitus, $\mathrm{CP}=$ constrictive pericarditis, $\mathrm{TBP}=$ tuberculous pericarditis. 


\section{Figure.2 Stages of tuberculous pericarditis}

This figure shows the pathophysiological process of tuberculous pericarditis and its final stage-constrictive tuberculous pericarditis.

\section{Figure.3 Basis of hemodynamic changes after constriction}

The decreasing pressure is restricted to be transmitted to the pericardial cavity through the intrathoracic when inhaling, and with a decrease of intrathoracic pressure. The constrictive pericardium makes the diastolic function impaired, and lead to a difference of pressure between left atrial and pulmonary vein, which causes a decreasing flow into the left ventricular. So we can see the shift of ventricular septum during the breathing. $\mathrm{LA}=$ left atrium $\mathrm{RA}=$ right atrium, $\mathrm{LV}=$ left ventricle, $\mathrm{RV}=$ right ventricle, $\mathrm{PV}=$ pulmonary vein, $\mathrm{P}=$ pressure.

\section{Figure.4 Diagnostic ideas of TBCP}

The diagnosis of TBCP is relatively difficult, here is a logic for diagnosis.

Table.1.Animal model of pericarditis or CP

\begin{tabular}{llll}
\hline Time & Author & Animal & Factor \\
\hline 1971 & Tsui CY, Burch GE[61] & mice & Coxsackie virus B4 \\
1980 & Matsumori A, Kawai C[62] & mice & Coxsackie virus B3 \\
1982 & W C. Reeves, et al[63] & rabbit & Radiation \\
1984 & N G. Pandian, et al[64] & dog & an irritant mixture consisting of tinct \\
1986 & Pagé PL[65] & dog & sterile talcum powder \\
1987 & L V, Leak, et al[66] & sheep & Heat-killed staphylococci and Freund' \\
2004 & Marina Afanasyeva, et al[67] & Wild-type and IFN- $\gamma$-KO BALB/c mice & Mice received subcutaneous injections \\
2007 & Andre d'Avila, et al[68] & swine & underwent epicardial mapping and ab \\
2009 & Panupong Jiamsripong, et al[69] & swine & ethyl cyanoacrylate glue with 8-12stit \\
2016 & L A Bokeriya, et al[70] & rabbit & Postoperative pericarditis in rabbits \\
\hline
\end{tabular}

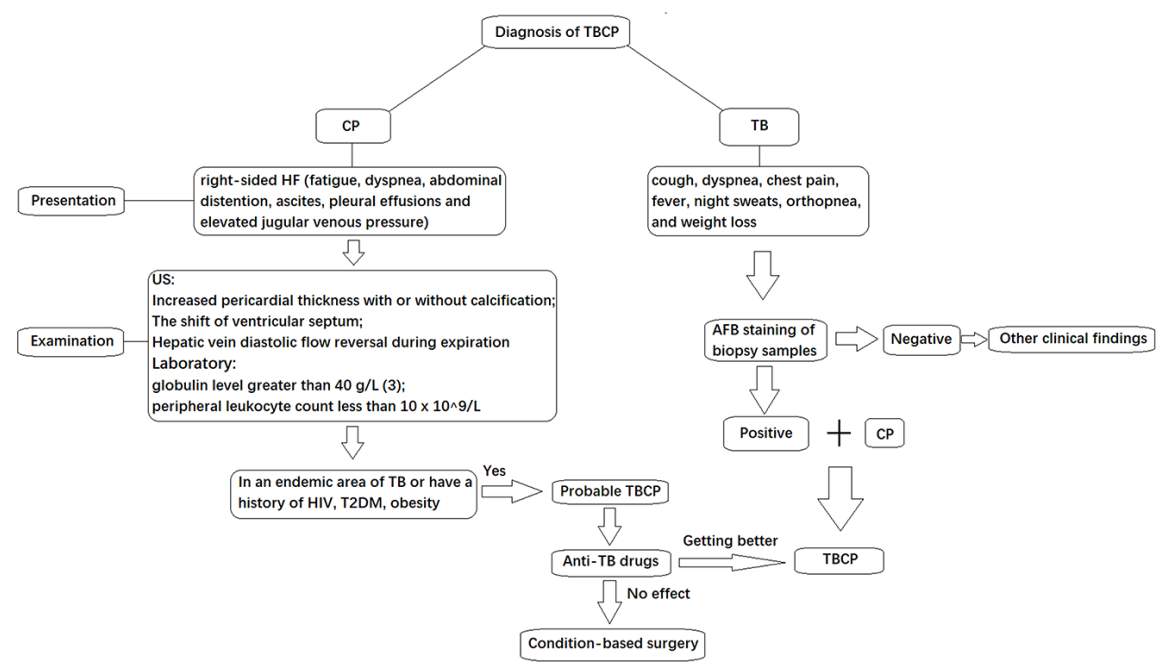



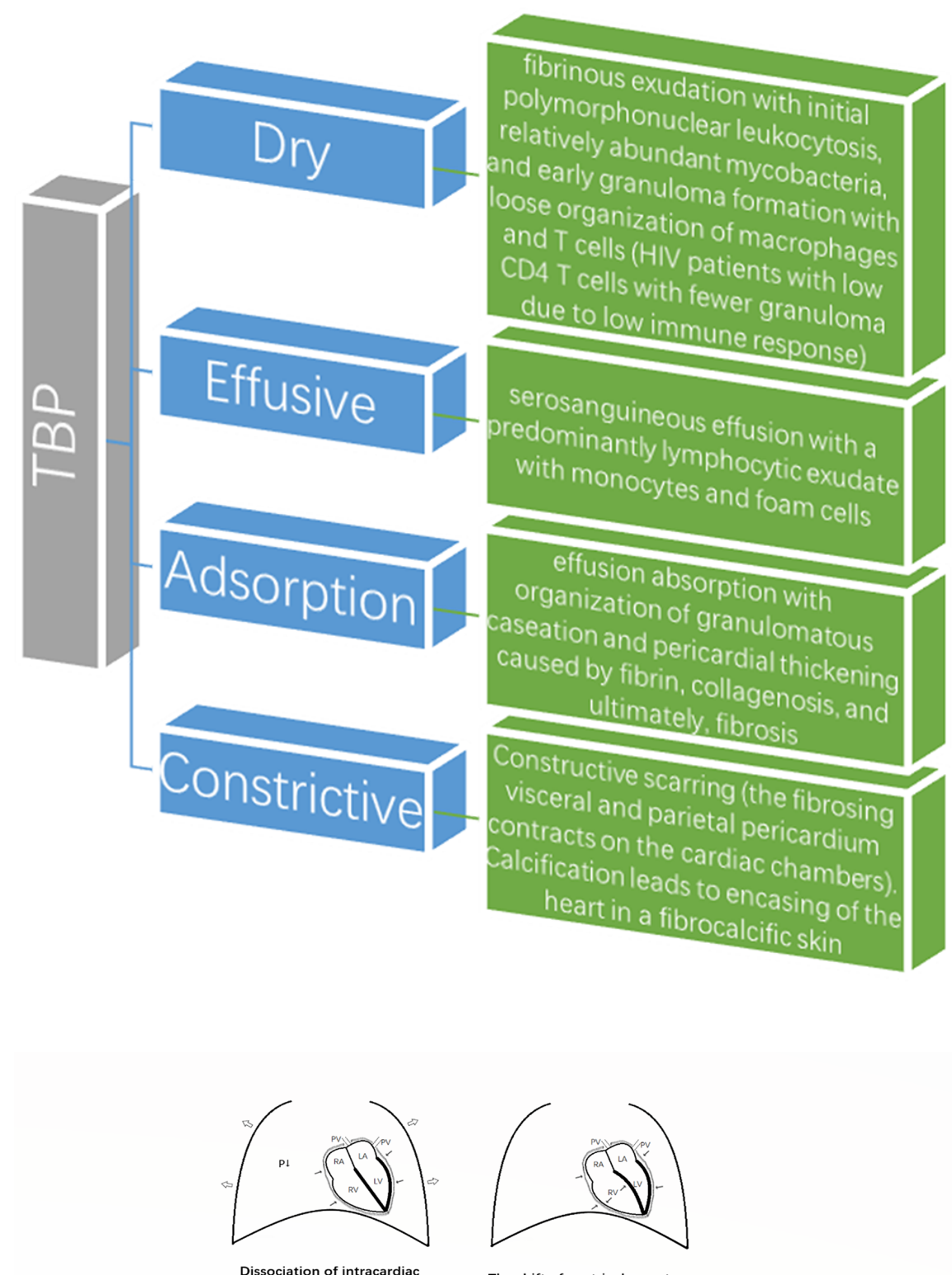

and intrathoracic pressur

The shift of ventricular septum during inspiration

caused by constriction 


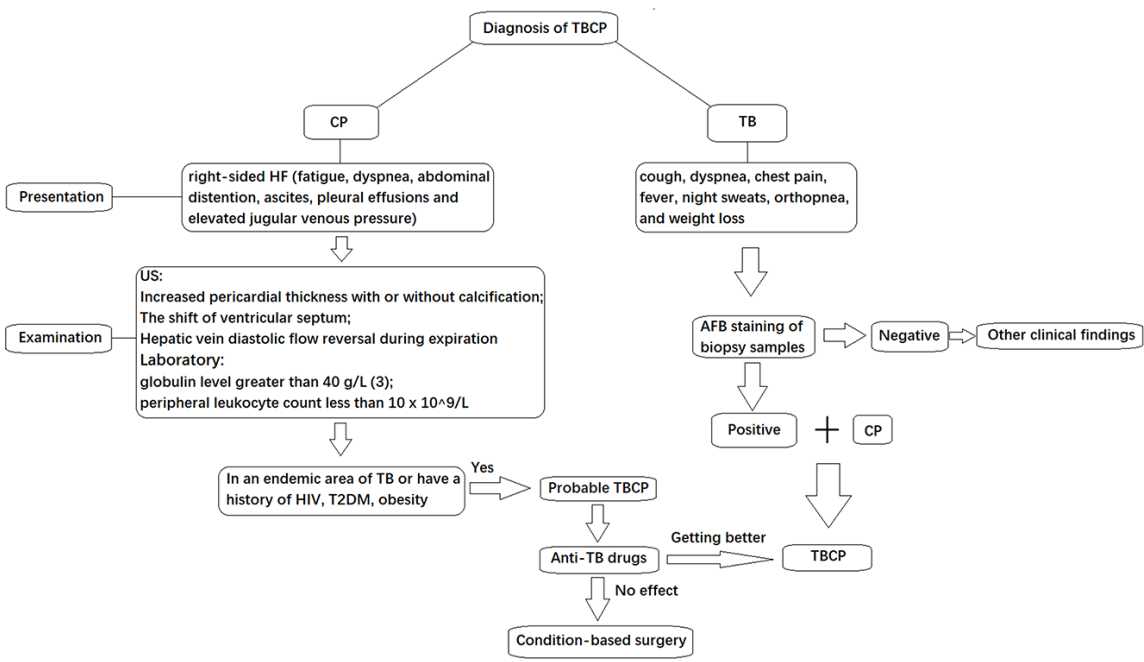

